ARTICLE

Received 9 Sep 2016 | Accepted 10 Feb 2017 | Published 28 Mar 2017 | Updated 11 Aug 2017 Dol: 10.1038/ncomms14881

OPEN

\title{
Stable and solubilized active Au atom clusters for selective epoxidation of cis-cyclooctene with molecular oxygen
}

Linping Qian ${ }^{1,2, \star}$, Zhen Wang ${ }^{2,3, \star}$, Evgeny V. Beletskiy², Jingyue Liu ${ }^{4}$ Haroldo J. dos Santos 2,5,6, Tiehu Li , Maria do C. Rangel ${ }^{5}$, Mayfair C. Kung ${ }^{2} \&$ Harold H. Kung ${ }^{2}$

The ability of Au catalysts to effect the challenging task of utilizing molecular oxygen for the selective epoxidation of cyclooctene is fascinating. Although supported nanometre-size $\mathrm{Au}$ particles are poorly active, here we show that solubilized atomic Au clusters, present in $\mathrm{ng} \mathrm{ml}^{-1}$ concentrations and stabilized by ligands derived from the oxidized hydrocarbon products, are active. They can be formed from various Au sources. They generate initiators and propagators to trigger the onset of the auto-oxidation reaction with an apparent turnover frequency of $440 \mathrm{~s}^{-1}$, and continue to generate additional initiators throughout the auto-oxidation cycle without direct participation in the cycle. Spectroscopic characterization suggests that 7-8 atom clusters are effective catalytically. Extension of work based on these understandings leads to the demonstration that these Au clusters are also effective in selective oxidation of cyclohexene, and that solubilized Pt clusters are also capable of generating initiators for cyclooctene epoxidation.

\footnotetext{
${ }^{1}$ Department of Chemistry and Shanghai Key Laboratory of Molecular Catalysis and Innovative Materials, Fudan University, Shanghai 200433, China.

${ }^{2}$ Chemical and Biological Engineering Department, Northwestern University, Evanston, Illinois 60208, USA. ${ }^{3}$ School of Materials Science and Engineering, Northwestern Polytechnical University, Xi'an, Shaanxi 710072, China. ${ }^{4}$ Physics Department, Arizona State University, Tempe, Arizona 85281, USA. ${ }^{5}$ Instituto de Química, Universidade Federal da Bahia, Campus Ondina, Federação, Salvador 40170-280, Bahia, Brazil. ${ }^{6}$ Instituto Federal de Educação, Ciência e Tecnológica da Bahia, Eunápolis 45820-970, Bahia, Brazil. * These authors contributed equally to this work. Correspondence and requests for materials should be addressed to M.C.K. (email: m-kung@northwestern.edu) or to H.H.K. (email: hkung@northwestern.edu).
} 
C urrent interest in noble metal atom clusters with dimensions less than the Fermi wavelength of conduction electrons arises from their distinctly different physical and chemical properties from those of single-atom species and bulk metal, and their potential to provide linkages between the materials at both ends of the size spectrum. They exhibit electronic quantum size and photochromic effects ${ }^{1}$, a high degree of structural fluxionality ${ }^{2-4}$, and cluster size-sensitive catalytic properties, for example, ref. 5. In addition to possessing fascinating properties, they also have broad applications in diverse fields such as sensors and bioimaging ${ }^{6}$. Consequently, significant effort has been expanded in the preparation and characterization of well-defined materials such as size-selected clusters $^{7}$ or ligand-capped monodisperse nanoclusters ${ }^{8}$. Colloidal preparation is also common, and the strongly bound surfactants are critical in determining the morphology and size of the colloidal particles ${ }^{9}$. Although effective in atom cluster preparation, strong metal-ligand interaction could influence the surface chemical properties of the clusters, especially their catalytic properties. Thus, it remains important to investigate properties of these atom clusters under conditions relevant to their applications, such as under catalytic reaction conditions.

Epoxides are precursors to many valuable chemicals, and their direct selective formation from olefins using $\mathrm{O}_{2}$ in a solvent-free reaction is environmentally friendly, highly desirable but challenging. Selective epoxidation of olefins using $\mathrm{O}_{2}$ is practiced only for ethylene and higher olefins that do not contain allylic hydrogen atoms ${ }^{10-12}$. For other olefins, expensive oxidants such as $\mathrm{H}_{2} \mathrm{O}_{2}$ or alkyperoxides are used. Thus, the seminal work of Lambert demonstrating the feasibility of converting styrene to its epoxide $\left(10-30 \%\right.$ selectivity) using $\mathrm{O}_{2}$ over supported $\mathrm{Au}$ catalysts derived from $\mathrm{Au}_{55}$ clusters generated considerable excitement ${ }^{13}$. Subsequently, the use of $\mathrm{O}_{2}$ to effect epoxidation of cyclic olefins and styrene has been explored for a diverse set of $\mathrm{Au}$ catalysts deposited on a variety of supports ${ }^{8,14-16}$, but the selectivities, except with cis-cyclooctene (COE) substrate, were poor. Even for COE, the oxidation activity was low ${ }^{17}$.

Improvement of the Au catalysts in these reactions necessitates an understanding of what constitutes the active catalyst. The examples mentioned above were all supported $\mathrm{Au}$ catalysts, yet there appears to be no consensus regarding the optimal particle size of $\mathrm{Au}^{8,13-15,17}$. The $\mathrm{Au}$ particles in these examples ranged from monodisperse atomic clusters ${ }^{8}$ to polydispersed $\mathrm{Au}$ nanoparticles spanning a broad size range of 20-150 nm (ref. 14). These catalysts were considered to be heterogeneous in nature and their apparent recyclability ${ }^{11,15}$ reinforced this impression. However, the reused catalysts were recovered by centrifugation $^{11,15}$, a procedure whereby solubilized Au species can be re-deposited onto the support. Thus, the seeming lack of consensus on the optimal Au particle size $8,13-15,17$ can be reconciled if we consider the catalysts as only a source of $\mathrm{Au}$ and the catalytic relevant species are solubilized $\mathrm{Au}$ atom clusters. Here we demonstrate the presence of solubilized, stable Au atom clusters containing less than 10 atoms that are generated in the reaction process and stabilized by the reaction medium. We demonstrate that they are active in initiating cyclooctene epoxidation with an apparent turnover frequency of $440 \mathrm{~s}^{-1}$. We further correlate the appearance of these atomic clusters with distinctive optical properties to the emergence of high catalytic activity and show that solubilized $\mathrm{Pt}$ atom clusters are also effective in initiating this reaction.

\section{Results}

Generation of solubilized Au clusters. Solvent-free oxidation of COE using $\mathrm{O}_{2}$ was conducted in the absence of light and either with or without COE stabilizer present. Removal of the COE stabilizer was achieved either by distillation or $\mathrm{KOH}$ treatment (see Supplementary Methods Section 1.1 for details), having in mind the recent report that oxidation of commercial cyclic alkenes using $\mathrm{O}_{2}$ over Au/graphite was only possible after removal of the added stabilizer ${ }^{17}$. The former method seemed to yield more consistent results. Using the $\mathrm{Au} / \mathrm{SiO}_{2}-\mathrm{A}$ catalyst, the stabilizer-free COE was oxidized to cyclooctane epoxide with high selectivity $(\geq 80 \%)$ after an induction period of $\sim 2 \mathrm{~h}$ (Fig. 1). The induction period was substantially longer $(>5 \mathrm{~h})$ for two other $\mathrm{Au} / \mathrm{SiO}_{2}$ preparations $\left(\mathrm{Au} / \mathrm{SiO}_{2}-\mathrm{B} 1\right.$ and -B2) of average $\mathrm{Au}$ particle sizes of 0.9 and $4.5 \mathrm{~nm}$, in which the $\mathrm{Au}$ particles were encapsulated by a nanoporous layer of $\mathrm{SiO}_{2}$ (Supplementary Fig. 1). Starting with $\mathrm{AuCl}$ or $\mathrm{AuCl}_{3}$, similar conversion-time profiles but with significantly longer induction periods were observed (Fig. 1). In these data shown for $\mathrm{AuCl}$, the stabilizer was not removed as its presence did not affect the induction period. The Au salts are poorly soluble in COE and the gas disperser and reaction vessel walls were partly coated with a $\mathrm{Au}$ film at the end of the reaction, likely formed from the decomposition and reduction of the unstable $\left(\text { cis- } \mathrm{C}_{8} \mathrm{H}_{14}\right)_{x} \mathrm{AuCl}_{y}$ complex ${ }^{18}$.

Despite the large differences in the induction periods, the product distributions using any of the Au sources were very similar (Supplementary Table 1), and the post-induction reaction rates were also similar to within a factor of 2 (Supplementary Table 2). In spite of the uncertainties in correction for evaporative losses, the carbon balance was good, better than $\pm 10 \%$ consistently even at $>70 \%$ conversion and as low as $\pm 1 \%$ at $<10 \%$ conversion. The predominant oxidation product was cyclooctane epoxide $(80 \%$ selectivity at $50 \%$ conversion, Supplementary Fig. 1 and Supplementary Table 1), followed by small amounts of 2-cycloocten-1-ol, 2-cycloocten-1-one and trace amounts of 1,2-cyclooctanediol. Small amounts of cyclooctene 3-hydroperoxide were detected by nuclear magnetic resonance $(\mathrm{NMR})^{19}$. A small amount of high molecular weight products, whose sticky nature prevented the use of electrospray ionization mass spectrometry to characterize the reaction mixture, was also present. As a control experiment, without adding any Au source, there was no reaction detected for stabilizer-free COE for over $10 \mathrm{~h}$, and slow reaction afterwards forming epoxide (curve d, Fig. 1). Thus, Au is effective in initiating the COE epoxidation reaction.

There is literature precedence of an induction period in cyclic alkene oxidation with $\mathrm{O}_{2}$. This was ascribed to the time needed

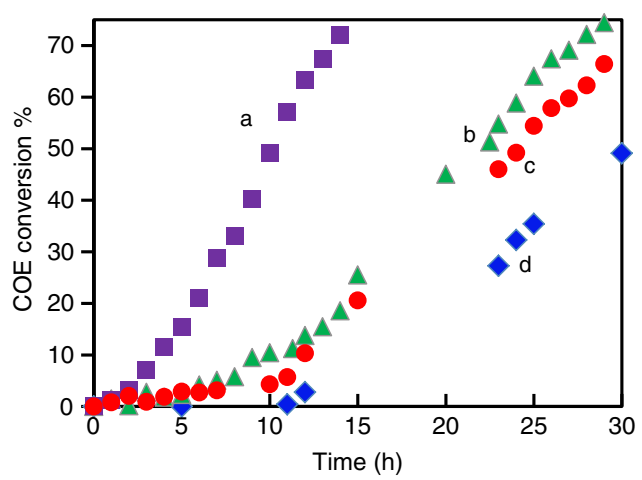

Figure 1 | Time course of cyclooctene conversion. $\mathrm{COE}$ conversion using $80 \mathrm{mg} \mathrm{Au} / \mathrm{SiO}_{2}-\mathrm{A}$ (a, square), $6 \mathrm{mg} \mathrm{AuCl}$ (b, triangle), $7 \mathrm{mg} \mathrm{AuCl} 3$ (c, circle), or no Au (d, diamond). Reaction conditions: $10 \mathrm{ml} \mathrm{COE}, 1 \mathrm{ml}$ decane, $\mathrm{O}_{2}$ bubbling rate $30 \mathrm{ml} \mathrm{min}^{-1}, 100^{\circ} \mathrm{C}$. For experiments with $\mathrm{Au} / \mathrm{SiO}_{2}$ or when no Au was present, $\mathrm{COE}$ was stabilizer free but, for experiments with $\mathrm{AuCl}$ and $\mathrm{AuCl}_{3}, \mathrm{COE}$ contained 100-200 p.p.m. Irganox 1,076 stabilizer. Conversion is defined based on initial COE present. 
for the supported $\mathrm{Au}$ particles to attain a sufficiently large size $(>2 \mathrm{~nm})$ for activity ${ }^{15}$. However, we observed that the reaction rates after the induction period were similar regardless of the duration of the induction period. At $50 \%$ conversion, the rates were 5.0, 3.9 and $3.5 \pm 0.2 \mathrm{mmolh}^{-1}$ for $\mathrm{Au} / \mathrm{SiO}_{2}-\mathrm{A}, \mathrm{Au} / \mathrm{SiO}_{2}-\mathrm{B} 2$, and $\mathrm{AuCl}$, respectively (Supplementary Table 2). These results suggested that the nature of the solid Au material is unimportant. In fact, the reaction proceeded equally well after the solid $\mathrm{Au}$ material was removed by filtration, vide infra. Thus, we propose that in the earliest phase of the induction period, a very small amount of hydrocarbon was oxidized and the oxygenated products served as ligands that extracted and solubilized active $\mathrm{Au}$ atom clusters into solution. These active $\mathrm{Au}$ atom clusters activated $\mathrm{O}_{2}$ to abstract $\mathrm{H}$ atoms from COE molecules and initiated the auto-oxidation phase of $\mathrm{COE}$ conversion to epoxide. Indeed, analysis of the reaction solution after hot filtration using a $0.2 \mu \mathrm{m}$ syringe filter to remove all solids showed low concentrations of solubilized Au typically from 10 to $200 \mathrm{ng} \mathrm{ml}^{-1}$, depending on the $\mathrm{Au}$ source and reaction condition. Although the filtration was conducted as rapidly as possible, some cooling of the hot liquid and possible precipitation in the syringe was unavoidable, although none was observed. The formation of stable Au clusters requires both solubilization of $\mathrm{Au}$ and generation of stabilizing ligands. Both processes require $\mathrm{O}_{2}$ and no reaction was detected when $\mathrm{O}_{2}$ was replaced by $\mathrm{N}_{2}$ in the gas stream. Similarly, formation of stable Au clusters can only be achieved in the presence of appropriate ligating molecules. Thus, the induction period was unchanged when $\mathrm{O}_{2}$ was bubbled though a suspension of Au precatalyst in decane before mixing in $\mathrm{COE}$.

That both oxygen and COE were necessary to generate solubilized $\mathrm{Au}$ suggests that the effective ligands that facilitate Au dissolution are oxygenated hydrocarbons. Addition of cyclooctanol, a molecule similar to the minor product cyclooctenol, to the reaction mixture did not affect the induction period or the reaction rate (Supplementary Fig. 3a). On the other hand, addition of cyclooctane-1,2-diol both shortened the induction period and enhanced the reaction rate, the extents of which increased with increasing concentration of diol added. Concurrently, the concentration of solubilized $\mathrm{Au}$ increased substantially (Supplementary Fig. 3b and Supplementary Table 3). Since the diol is oxidized readily to carboxylic acid under the reaction conditions, as determined by NMR, it appears that diacids were effective chelating ligands in stabilizing $\mathrm{Au}$ clusters.

Catalytic role of solubilized Au clusters. Aliquots of reactionderived solution obtained by hot filtration were active for initiation of epoxidation of fresh $\mathrm{COE}$, and the activity was stable for weeks when stored in the dark at room temperature. The reaction profile using a filtrate was similar to that of the original mixture but with an important distinction that the induction period was absent (Supplementary Fig. 4). Whether the reaction was initiated with an aliquot of filtrate or solid $\mathrm{Au}$ precatalysts, the reaction rate beyond $\sim 20 \%$ conversion was similar (Supplementary Fig. 4) and significantly higher than those at low conversions. This phenomenon is consistent with the auto-oxidation nature of this phase of reaction.

After initiation, auto-oxidation of hydrocarbon is propagated by reactive intermediates such as hydroperoxide and other free radicals. In all reactions terminated at conversions $>60 \%$, hydroperoxide was detected by titration at concentrations equivalent to $\sim 2 \%$ of the initial $\mathrm{COE}$ concentration ( $\sim 2 \%$ yield). In one experiment using $\mathrm{AuCl}$ as the $\mathrm{Au}$ source, the concentration of hydroperoxide was monitored throughout the reaction and found to be equivalent to a yield of 0.9 at $28 \%$ conversion, 1.5 at $47 \%$ conversion and $\sim 2.0 \%$ at conversions $60 \%$ and higher.

Together with solubilized $\mathrm{Au}$, hydroperoxide and reactive intermediates in the reaction-derived filtrate contributed to the initiation of COE epoxidation and the elimination of the induction period. To differentiate and quantify the contribution of solubilized $\mathrm{Au}$ clusters to the initial reaction rate, the following experiments were conducted. Since it is known that triphenylphosphine $\left(\mathrm{PPh}_{3}\right)$ reacts quantitatively and converts peroxide to the corresponding alcohol ${ }^{20}$, we first tested whether $\mathrm{PPh}_{3}$ was able to quantitatively suppress the epoxidation reaction. Thus, a COE epoxidation reaction was initiated using tert-butyl peroxide $(t-\mathrm{BuOOH})$ without any $\mathrm{Au}$ addition. After reaching $77 \%$ conversion (Supplementary Fig. 5), the solution was hot-filtered for procedural consistency although there were no solids. This stock filtrate obtained contained 2.95 mmoles of hydroperoxide, which was probably cyclooctene 3-hydroperoxide as the original $t$ - $\mathrm{BuOOH}$ should have been consumed completely. A $2 \mathrm{ml}$ aliquot of this stock filtrate was used to initiate another COE reaction (Fig. 2, curve c), and the initial rate was $0.75 \pm 0.25 \mathrm{mmoles}^{-1}$. To another $2 \mathrm{ml}$ of the same stock filtrate, 1.05 equivalent $\mathrm{PPh}_{3}$ was added, and the mixture was used to initiate another COE reaction. No peroxide was detected after the addition of $\mathrm{PPh}_{3}$ and concomitantly, the initial activity was completely suppressed (Fig. 2, curve d). Thus $\mathrm{PPh}_{3}$ was effective in consuming free radical initiators of the auto-oxidation reaction. These serve as control experiments for Au-catalysed initiation experiments.

A stock solution of a $\mathrm{Au} / \mathrm{SiO}_{2}-\mathrm{A}$ derived filtrate was first prepared. Then $2 \mathrm{ml}$ of this stock solution was used to initiate a COE epoxidation reaction, and the initial rate was found to be $2.7 \pm 0.25 \mathrm{mmol} \mathrm{h}^{-1}$ (Fig. 2, curve a). Next, another $2 \mathrm{ml}$ of the same stock solution was mixed with 1.05 eq. $\mathrm{PPh}_{3}$ (with respect to hydroperoxide detected). This mixture, with no detectable hydroperoxide, was used to initiate another COE reaction. The initial rate was $1.8 \pm 0.25 \mathrm{mmolh}^{-1}$ (Fig. 2, curve b), and the time course of product distribution was similar to that without $\mathrm{PPh}_{3}$ addition (Supplementary Fig. 6). Thus, Au contributed $1.8 \mathrm{mmol} \mathrm{h}^{-1}$ to the initial COE epoxidation rate, corresponding to a turnover frequency (TOF) of $440 \mathrm{~s}^{-1}$ based on total

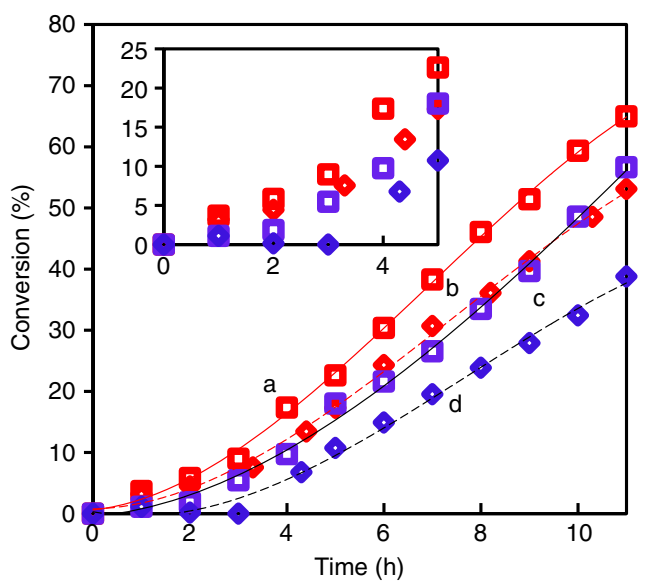

Figure 2 | Effect of triphenylphosphine on reaction rates. Effect of triphenylphosphine $\left(\mathrm{PPh}_{3}\right)$ addition on the initial rates of $\mathrm{COE}$ oxidation. Curves $a, b$ : fivefold diluted filtrates obtained from COE oxidation using $\mathrm{Au}-\mathrm{SiO}_{2}-\mathrm{A} ; \mathrm{Au}=20 \mathrm{ng} \mathrm{ml}^{-1}$ and hydroperoxide $=0.29$ mmoles. Curve a: filtrate, b: filtrate +0.30 mmoles $\mathrm{PPh}_{3}$. Curves c, d: fivefold diluted filtrates obtained from $\mathrm{COE}$ oxidation using $t-\mathrm{BuOOH}$ as reaction initiator. c: filtrate and d: filtrate $+0.62 \mathrm{mmoles}^{\mathrm{PPh}}$. Inset uses the same symbols. 
solubilized $\mathrm{Au}$ present. The difference between the two rates $\left(0.9 \mathrm{mmol} \mathrm{h}^{-1}\right)$ was ascribed to contributions from hydroperoxide and other reactive intermediates.

The conclusions from these experiments were substantiated using other sets of stock filtrates. As shown by Exp. 1 in Supplementary Table 4, after removal of hydroperoxide with $\mathrm{PPh}_{3}$, the stock Au filtrate retained significant activity to initiate the reaction, whereas the stock $t-\mathrm{BuOOH}$ filtrate lost its activity (Exp. 3).

An additional experiment was conducted to probe the effect of solubilized $\mathrm{Au}$ concentration on the initiation rate (Exp. 2 in Supplementary Table 4). Starting with a stock Au filtrate, a series of three consecutive runs was performed, in which $2 \mathrm{ml}$ aliquot of the filtrate of the preceding run was used as initiator. Thus, at the start of each subsequent run in the series, the organic reactive intermediate would be relatively similar, but the solubilized Au would be repeatedly diluted, such that its concentration in Exp. 1 was 5.4 times more concentrated than the next run, and 29 times more concentrated than Exp. 2 (which was 157 times more dilute than the stock filtrate). In addition, 1 eq. of $\mathrm{PPh}_{3}$ corresponding to the hydroperoxide present in the aliquot was added to Exps 1 and 2 at the beginning of the runs. The initial rate observed for Exp. 2 decreased by $\sim 25$ times compared with Exp. 1 (Supplementary Table 4). This very low initial rate in run with highly diluted solubilized $\mathrm{Au}$ is in line with experiments using stock $t$ - $\mathrm{BuOOH}$ filtrate with $\mathrm{PPh}_{3}$ addition, where there were no $\mathrm{Au}$ and the initial activity was not measurable.

Another series of experiments was performed to probe the effect of solubilized $\mathrm{Au}$ concentration on the rate of autooxidation. In these experiments (Fig. 3), a standard COE reaction using $\mathrm{Au} / \mathrm{SiO}_{2}-\mathrm{A}$ as the Au source was conducted first (Run 1). Then $2 \mathrm{ml}$ of its filtrate was used to initiate Run 2. Afterwards, $2 \mathrm{ml}$ of the solution from Run 2 was used to initiate Run 3. As before, the concentrations of organic hydroperoxide and reactive intermediates were similar at the beginning of Runs 2 and 3, but the concentration of solubilized $\mathrm{Au}$ in Run 3 was 5.4 times lower than Run 2, and 29 times lower than Run 1. No induction period was observed in either Run 2 or 3 as observed earlier, and the reaction rates at $50 \%$ conversion decreased from $5.3 \pm 0.2 \mathrm{mmolh}^{-1}$ in Run 1 , to $4.6 \pm 0.2 \mathrm{mmol} \mathrm{h}^{-1}$ in Run 2, and $3.5 \pm 0.2 \mathrm{mmol} \mathrm{h}^{-1}$ in Run 3 (Supplementary Table 5). This small but consistent decrease in rate with increasing dilution of
Au was observed reproducibly, and is consistent with an autooxidation mechanism that does not depend on $\mathrm{Au}$, and that the function of $\mathrm{Au}$ is to initiate the reaction. The slight decrease in reaction rate with each subsequent dilution of $\mathrm{Au}$ indicated that, although not a catalyst in the auto-oxidation cycle, the solubilized $\mathrm{Au}$ continued to contribute to the $\mathrm{COE}$ conversion by generating initiators for the auto-oxidation cycle. As the concentration of $\mathrm{Au}$ decreased with dilution, this contribution diminished, and the auto-oxidation reaction rate declined. For the experiment under consideration, there was $6 \mathrm{nmol}$ solubilized $\mathrm{Au}$ in the original solution. If we assume that after 29 -fold dilution, the contribution of solubilized $\mathrm{Au}$ to the auto-oxidation reaction became negligible, then the solubilized $\mathrm{Au}$ was contributing $0.33 \pm 0.2 \mathrm{mmol}(\mathrm{nmol} \mathrm{Au} \cdot \mathrm{h})^{-1}$ to the auto-oxidation reaction by injecting initiators at $50 \%$ conversion, or an apparent TOF of $92 \mathrm{~s}^{-1}$.

Nature of the solubilized Au clusters. The filtrate from a reaction using the $\mathrm{Au} / \mathrm{SiO}_{2}-\mathrm{A}$ as the $\mathrm{Au}$ source was examined with aberration-corrected transmission electron microscopy (TEM; Fig. 4). The images showed Au species present mainly as small clusters up to $\sim 0.7 \mathrm{~nm}$, including single Au atoms. A few larger clusters were found occasionally. Supplementary Fig. 7 shows the size distribution of the Au clusters.

These filtrates were also examined using fluorescence spectroscopy. The filtrate from reaction using the $\mathrm{Au} / \mathrm{SiO}_{2}-\mathrm{A}$ source showed a broad emission peak at $\sim 450 \mathrm{~nm}$ with a corresponding excitation peak at $\sim 405 \mathrm{~nm}$ (Fig. 5, curves a, $\mathrm{a}^{\prime}$ ), which were not observed without $\mathrm{Au}$ (that is, not present in the reaction mixture derived from $t$ - $\mathrm{BuOOH}$ initiator, Supplementary Fig. 8). Similar spectra were observed with filtrates derived from $\mathrm{AuCl}$ (Supplementary Fig. 9) and AuCl-diol combination. For the latter, the concentration of solubilized $\mathrm{Au}$ increased with diol/Au ratio, and the intensities of both emission and excitation spectra increased simultaneously (Supplementary Fig. 10).

Small $\mathrm{Au}$ atomic clusters are known to exhibit discrete absorption and fluorescence, and the emission maxima have been shown to scale with the cluster size as predicted by the spherical Jellium model ${ }^{21}$. On the basis of this model, these spectra indicated the presence of predominantly eight-atom clusters (Supplementary Table 6). These emission peaks were not
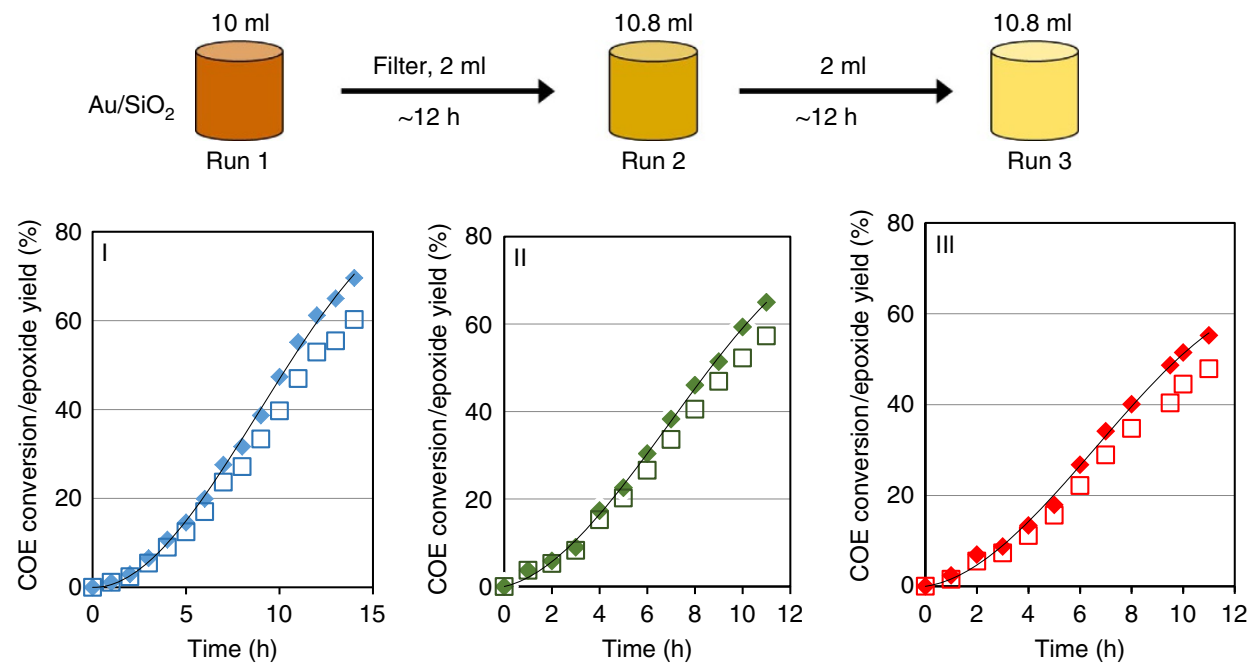

Figure 3 | Cyclooctene conversions in consecutive experiments. Experiments of repeated use of filtrate from a $\mathrm{COE}$ epoxidation run using $\mathrm{Au} / \mathrm{SiO}_{2}-\mathrm{A}$ as Au source. Run 1 (panel I) was a reaction similar to those in Fig. 1. Afterwards, the mixture was hot-filtered. 2 ml of filtrate from Run 1 was used for Run 2 by adding COE and an internal standard of decane to $10.8 \mathrm{ml}$ total volume (panel II), and $2 \mathrm{ml}$ of the reaction mixture of Run 2 was mixed with fresh COE/decane for Run 3 (panel III). 
detected during the induction period but was prominent when the catalytic activity was high (Supplementary Fig. 11). This suggests that they are related to the catalytic activity. Also consistent with their catalytic relevance was the observation that, in general, solutions that showed high activities for COE epoxidation also exhibited highly intense peaks (Supplementary Fig. 10).

The emission peaks of $\mathrm{Au} / \mathrm{SiO}_{2}$-A derived filtrate shifted to $\sim 425$ and $375 \mathrm{~nm}$ upon 12- and 120-fold dilution with ethanol, respectively, and the corresponding excitation peak maxima to $\sim 360$ and $310 \mathrm{~nm}$ (Fig. 5a, spectra b and c). Their intensities decreased with increasing dilution. Blue-shifts of peaks by about the same amount were also observed when diluted with a non-coordinating, nonpolar solvent like COE (Supplementary Fig. 12). Likewise, dilution of filtrates derived from $\mathrm{AuCl}$ with ethanol also resulted in peak shifts to shorter wavelengths (Supplementary Fig. 9a). The similar extents of blue-shift with dilution with ethanol and $\mathrm{COE}$ suggested that it was not due to solvent effect. Instead it was due to changes in the Au cluster size in response to the changed $\mathrm{Au}$ and ligand concentrations of the solution.

The broad spectral bandwidth of the filtrate emission peaks (Fig. 5a, curve a) suggests that they are composites of overlapping emission peaks from $\mathrm{Au}$ clusters of different sizes. This was
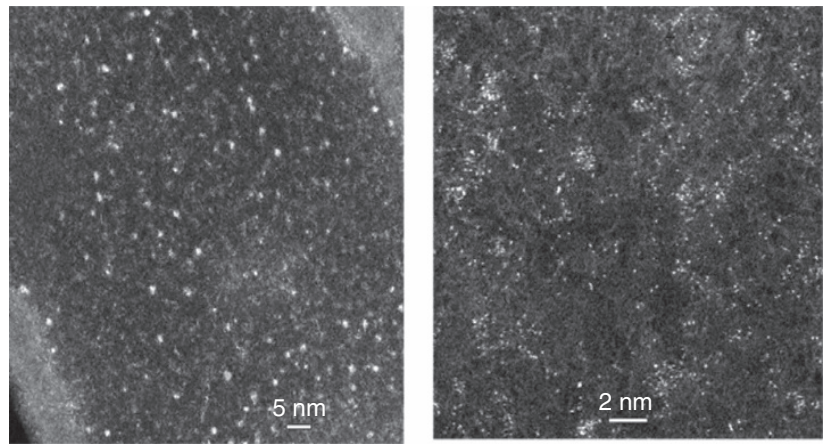

Figure 4 | Aberration-corrected electron microscopy images of filtrates. TEM images of filtrate from $\mathrm{Au} / \mathrm{SiO}_{2}-\mathrm{A}$ collected after conversion reached $18 \%$. The filtrate was diluted with ethanol and dried on the grid. Scale bar, $5 \mathrm{~nm}$ and $2 \mathrm{~nm}$ for the left and right images.

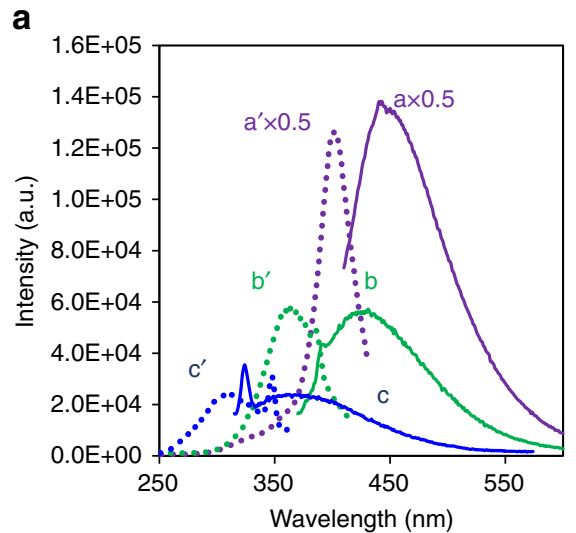

confirmed by the interesting dependence of intensity with excitation wavelength for solutions of different Au concentrations as is shown in Fig. $5 \mathrm{~b}$ for $\mathrm{Au} / \mathrm{SiO}_{2}-\mathrm{A}$ derived filtrate and Supplementary Fig. $9 \mathrm{~b}$ for $\mathrm{AuCl}$ derived filtrate. Figure $5 \mathrm{~b}$ compares the emission spectra obtained with 400 and $360 \mathrm{~nm}$ excitation of a filtrate and its 12 -fold diluted solution. With $400 \mathrm{~nm}$ excitation, the peak intensity of the filtrate was higher than the 12 -fold diluted sample. With $360 \mathrm{~nm}$ light excitation, the reverse was observed-the emission peak of the 12 -fold diluted sample was more intense than the undiluted filtrate. Correspondingly, the absorption at $360 \mathrm{~nm}$ was more intense for the diluted sample (Fig. 5b, inset). The reversal in emission peak intensities for the original and diluted filtrates upon switching from 400 to $360 \mathrm{~nm}$ excitation can be interpreted to be due to a larger population of smaller clusters in the diluted sample, and smaller $\mathrm{Au}$ clusters are known to be more efficient in photoemission at shorter wavelengths ${ }^{21}$. The solubilized $\mathrm{Au}$ species are in equilibrium with each other in the solution, including those not detected by fluorescence but clearly discernable in aberrationcorrected TEM images (for example, $\mathrm{Au}$ atoms). Dilution decreased the concentrations of both $\mathrm{Au}$ and coordinating ligands, and affected the equilibrium size distribution of $\mathrm{Au}$ clusters.

Extension to other systems. It is possible that the function of solubilized $\mathrm{Au}$ to inject initiator/propagators into the autooxidation reaction can be extended to other olefins, and be replaced by other metals. Both of these were tested. $\mathrm{PtCl}_{4}$ was tested using the same procedure as for $\mathrm{AuCl}$, and the results are show in Supplementary Fig. 13. The reaction profile was very similar to using $\mathrm{Au}$; there was an induction period after which effective, selective epoxidation occurred. At the end of the reaction, the mixture was filtered hot, and the filtrate was used to initiate COE epoxidation without an induction period (Supplementary Fig. 13). The filtrate was found to have $0.1 \mathrm{mg} \mathrm{ml}^{-1}$ of solubilized $\mathrm{Pt}$, and it exhibited an emission spectrum (Supplementary Fig. 14). Thus, solubilized Pt can function like Au.

The filtrate derived from an Au-catalysed COE reaction and with hydroperoxide removed by $\mathrm{PPh}_{3}$ was tested for cyclohexene oxidation at $60^{\circ} \mathrm{C}$ using molecular $\mathrm{O}_{2}$. After $6 \mathrm{~h}, \sim 20 \%$ of

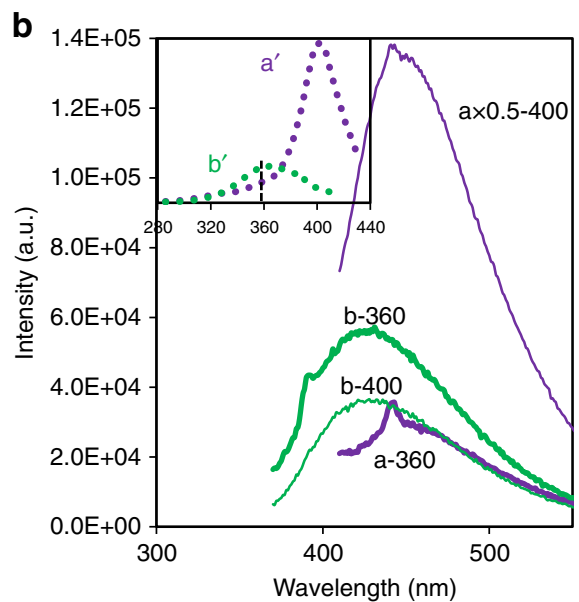

Figure 5 | Ultraviolet-visible excitation and emission spectra. (a) Emission (solid lines) and excitation (dotted lines) spectra of: (a,a') filtrate derived from $\mathrm{Au} / \mathrm{SiO}_{2}-\mathrm{A}$ (scaled by 0.5 ); (b, $\left.b^{\prime}\right)$ 12-fold ethanol-diluted filtrate; and $\left(c, c^{\prime}\right)$ 120-fold ethanol diluted filtrate. The parent filtrate contained $108 \mathrm{ng} \mathrm{ml}^{-1}$ of $\mathrm{Au}$. Emission spectra a-c were collected using $\lambda_{\text {excitation }}=405,360$, and $310 \mathrm{~nm}$, respectively. Excitation spectra $\mathrm{a}^{\prime}-\mathrm{c}^{\prime}$ were collected by monitoring at $\lambda_{\text {emission }}=450,425$ and, $375 \mathrm{~nm}$, respectively. The sharp features in spectra c, $\mathrm{c}^{\prime}$ are instrument artefacts (Supplementary Fig. 15). (b) Emission spectra of filtrate (a) and 12-times diluted filtrate (b) collected at $\lambda_{\text {excitation }}=400$ ( -400 curves) and $360 \mathrm{~nm}$ ( -360 curves). Inset showed the excitation spectra of the filtrates. 
cyclohexene was oxidized and the major product was cyclohexene hydroperoixde $(\sim 60 \%)$, with minor amounts of 2-cyclohexen-1ol, 2-cyclohexen-1-none, and cyclohexane epoxide, a distribution similar to that from auto-oxidation ${ }^{22,23}$. Without the Au-derived filtrate, there was no reaction. Thus, the solubilized $\mathrm{Au}$ was capable of initiating oxidation of other cyclic alkenes.

\section{Discussion}

We have demonstrated definitively that solubilized Au, derived from the $\mathrm{Au}$ nanoparticles on a support or Au salts, is the catalytic relevant species in COE epoxidation. The Au nanoparticles and $\mathrm{Au}$ salts are precatalysts and their roles comprised of serving as a source of $\mathrm{Au}$ as well as the producer of a minute number of stabilizing ligands for the solubilized $\mathrm{Au}$ at the earliest phase of the induction period. The formation rate of the initial solubilized $\mathrm{Au}$ clusters depends on the ease of dissolution of $\mathrm{Au}$ from its source and the ability of the $\mathrm{Au}$ source to catalyse the oxidation of COE into effective chelating oxygenated hydrocarbons. Thus, the induction period can be shortened by the addition of effective chelates, such as diols and molecules with multiple functional groups.

Figure 6 shows the manner in which the solubilized $\mathrm{Au}$ atom clusters interface with the auto-oxidation cycle (which is represented by a generic cycle since its details are not important for the present discussion). Au accelerates COE oxidation in two ways. In the initial phase, they play the essential role of producing initiators for the auto-oxidation phase of the COE reaction, which in turn generates more oxygenated ligands to form additional atomic clusters. The high activity of Au clusters in this phase was determined in filtrates with the organic hydroperoxide removed by $\mathrm{PPh}_{3}$ to be equivalent to a TOF of $440 \mathrm{~s}^{-1}$. In the auto-oxidation phase, these solubilized Au clusters continue to generate additional initiators to accelerate the auto-oxidation cycle. By comparing the rates of this phase of the reaction in experiments where a filtrate was used repeatedly through multiple cycles of dilution and thus decreasing $\mathrm{Au}$ contribution, the contribution of $\mathrm{Au}$ to the TOF at $50 \%$ conversion was $92 \mathrm{~s}^{-1}$. The ability of solubilized Au to continuously generate initiators distinguishes it from free radical initiators such as $t$-butyl hydroperoxide that are consumed in the initiation process. In this phase, the data also showed that the solubilized Au clusters do not participate in the auto-oxidation cycle directly, that is, $\mathrm{Au}$ is not a necessary component for auto-oxidation. It should be noted that if this point was not recognized and all the epoxidation activity was attributed to solubilized $\mathrm{Au}$, the TOF would mistakenly become $\sim 1,500 \mathrm{~s}^{-1}$ for this set of data. Since the auto-oxidation was rather insensitive to the concentration of $\mathrm{Au}$ (within a factor of two for [Au] ranging from 20 to $120 \mathrm{ng} \mathrm{ml}^{-1}$ ),

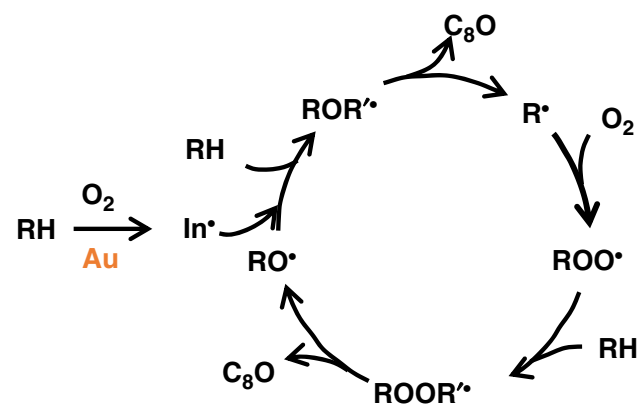

Figure 6 | Proposed function of $\mathrm{Au}$ in cyclooctene epoxidation with $\mathrm{O}_{2}$. Proposed catalytic function of $\mathrm{Au}$ atomic clusters in the autooxidation cycle for the formation of cyclooctene epoxide $\left(\mathrm{C}_{8} \mathrm{O}\right) . \mathrm{R}=\mathrm{c}-\mathrm{C}_{8} \mathrm{H}_{13}, \mathrm{R}^{\prime}=\mathrm{c}-\mathrm{C}_{8} \mathrm{H}_{14}$, and $I n \bullet$ is free radical initiator. Note that $A u$ functions to generate free radical initiators and is not necessary in the $\mathrm{C}_{8} \mathrm{O}$ formation cycle. an inflated value of TOF could reach as high as $7,000 \mathrm{~s}^{-1}$. For reactions that involve complex radical chain mechanism such as cyclohexane oxidation, often the contributions to the rate by $\mathrm{Au}$ catalyst and auto-oxidation are not separated but attributed entirely to $\mathrm{Au}$, resulting in over-estimated values of $\mathrm{TOFs}^{24,25}$.

This understanding of the role of solubilized Au resolves the controversy about the nature of the active form of supported $\mathrm{Au}$ catalysts for this reaction. In fact, such controversy can be found also for other Au-catalysed liquid phase oxidation reactions. For example, $\mathrm{Au} / \mathrm{C}$ catalysts with large $\mathrm{Au}$ particles are active for selective hydrocarbon oxidation ${ }^{17}$, whereas small $\mathrm{Au}$ atom clusters are reported to be the active form for thiophenol oxidation $^{26}$ and stilbene epoxidation ${ }^{27}$. An induction period is common for these reactions, yet few conducted hot filtration and other characterization to determine if a phenomenon similar to those observed here may occur. Thus further investigation is warranted.

The ability of $\mathrm{Au}$ to generate initiator is applicable to other inorganic initiators. Indeed, we showed that this can be accomplished with solubilized $\mathrm{Pt}$ formed from $\mathrm{PtCl}_{4}$. The amount of solubilized metal atom clusters (of the order of $\mathrm{ng} \mathrm{ml}^{-1}$ ) needed as catalyst initiators is significantly less than traditional catalysts employed for this class of reactions. Consequently, the very low costs associated with catalyst material and oxidant $\left(\mathrm{O}_{2}\right)$ enhance the appeal of this process for further development. From another angle, the fact that solubilized $\mathrm{Au}$ is effective in initiating other selective oxidation reactions such as cyclohexene oxidation with $\mathrm{O}_{2}$ broadens its potential field of application. There is a high probability that it is also effective in initiating other hydrocarbon oxidation reactions that are susceptible to free radical initiation, such as cyclohexane. The transferability of the formation and catalytic role of solubilized metal atom clusters to other systems offers exciting opportunities.

The solubilized $\mathrm{Au}$ is present as a mixture of species ranging from $\mathrm{Au}$ atoms to clusters of $\sim 0.7 \mathrm{~nm}$. The most abundant ones at the end of a reaction are 7-8 atom clusters judging from the positions $(\sim 450 \mathrm{~nm})$ and intensities of the emission peaks. The optical spectra of these clusters are absent during the induction period but are intense at the high-activity phase of the reaction, suggesting their catalytic role. In line with their catalytic role is that in different reaction solutions, the intensity of the $450 \mathrm{~nm}$ emission peak trended with the rates of COE conversions.

\section{Methods}

General information. Except otherwise noted, all chemicals were purchased and used without purification. Additional details of their sources and experimental procedures are in the Supplementary Section 1 Methods and Material. When applied, the stabilizer in COE was removed by distillation.

Catalysts. $\mathrm{Au} / \mathrm{SiO}_{2}-\mathrm{A}$ ( $2 \mathrm{~nm}$ average $\mathrm{Au}$ particle size) was prepared using $\mathrm{Au}(\mathrm{en})_{2} \mathrm{Cl}_{3}$ precursor, which was prepared by adding ethylenediamine (en, en $/ \mathrm{Au}=2.65$ ) to a water/ethanol solution of $\mathrm{HAuCl}_{4} \cdot 3 \mathrm{H}_{2} \mathrm{O}$, similar to the procedure of Zhu et al. ${ }^{28}$. After filtering, enough of this filtrate at $4.2 \mathrm{mM}$ was added to fume silica at $40^{\circ} \mathrm{C}$ for a nominal Au loading of $2.2 \mathrm{wt} . \%$. The $\mathrm{pH}$ of the solution was adjusted to 9 by dropwise addition of $0.75 \mathrm{M}$ en solution. The mixture was stirred for $2 \mathrm{~h}$ at room temperature, filtered, washed first with water at room temperature, and then with $40^{\circ} \mathrm{C}$ water. After calcination in a flowing $\mathrm{O}_{2} / \mathrm{O}_{3}$ mixture at $150^{\circ} \mathrm{C}$. the resulting powder was light yellow in colour, and had a $\mathrm{Au}$ loading of $1.2 \mathrm{wt} . \%$

$\mathrm{Au} / \mathrm{SiO}_{2}-\mathrm{B} 1$ (average $0.9 \mathrm{~nm}$ Au particles) and $-\mathrm{B} 2(4.5 \mathrm{~nm})$, in which the $\mathrm{Au}$ particles were partially encapsulated by thin islands of $\mathrm{SiO}_{2}$, were synthesized similar to our previously published method ${ }^{29}$. Briefly, poly(methylhydro)siloxane with trimethylsiloxy termination (PMHS) was partially oxidized ( $\sim 50 \%)$ with water over $\mathrm{Pd} /$ carbon catalysts. The resulting silanol groups were further reacted with N-methyl-aza-2,2,4-trimethylsilacyclopentane to form amine functionalized PMHS. Then $\mathrm{Au}(\mathrm{THT}) \mathrm{Cl}$ was added to the amine functionalized PMHS (amine/Au $=10$ ) and stirred for $0.5 \mathrm{~h}$ before the introduction of fumed silica. The mixture was filtered and washed, and the solid was calcined in a flowing $\mathrm{O}_{2} / \mathrm{O}_{3}$ mixture at $150^{\circ} \mathrm{C}$. The final Au weight loading of the sample was $0.9 \mathrm{wt} . \%$. The two samples differed in the degree of washing and dryness before calcination. 
Characterization. Emission and excitation spectra of the filtrates were recorded using Photon Technology International Model QM-2. An emission spectrum was collected by first determining the wavelength of the excitation light that would yield the maximum emission intensity. Once determined, the emission spectrum was collected using this excitation wavelength. Excitation spectrum was generated by monitoring the fluorescence emission at the wavelength of the emission maximum while varying the excitation wavelength. High-performance liquid chromatography grade tetrahydrofuran (THF) or ethanol was used for sample dilution when used. Inductively coupled plasma mass spectrometry was performed with ThermoiCAP Q Inductively Coupled Plasma Mass Spectrometry. Water was used to determine instrument artefacts (Supplementary Table 7), and background emissions due to dissolved organics and solvents were also measured (Supplementary Fig. 15). For preparation, $1 \mathrm{ml}$ of the sample was heated to $850^{\circ} \mathrm{C}$ to remove all organics. The remaining solid was dissolved in concentrated $\mathrm{HNO}_{3} / \mathrm{HCl}$, and diluted with water. High-angle annular dark-field scanning transmission electron microscopy images were obtained on a JEOL ARM-200F aberration-corrected STEM (AC-STEM) operated at $200 \mathrm{kV}$ with a nominal spatial resolution of $0.08 \mathrm{~nm}$ in the STEM mode. The TEM/STEM samples were prepared by dipping the lacey carbon covered copper TEM grid directly into ethanol-diluted filtrate solutions. After drying, the samples formed a thin film that covered the holes of the lacey carbon film.

Catalytic tests. Because of Au plating and oxidation activity of residual reaction liquids, it was essential that the reactor vessel was thoroughly cleaned after each use. An elaborate procedure as described in Supplementary Information was employed (Supplementary Methods Section 1.4.4), such that without adding a Au source, there was no reaction of COE for at least $10 \mathrm{~h}$. The catalytic oxidation of COE was conducted in the absence of light in a $50 \mathrm{ml}$ three-necked round bottom flask equipped with a fine grit glass gas disperser for bubbling $\mathrm{O}_{2}$ through the liquid and a condenser maintained at $-5^{\circ} \mathrm{C}$ (Supplementary Fig. 16). In a typical reaction, $10 \mathrm{ml}$ of COE, $1 \mathrm{ml} \mathrm{n}$-decane (internal standard), Au source and a Teflon-coated magnetic stirrer were loaded into the flask. The mixture was sonicated for $10 \mathrm{~min}$ in the dark, and then placed in an oil bath preheated at $100^{\circ} \mathrm{C}$ with stirring. After the mixture reached $100{ }^{\circ} \mathrm{C}$, the reaction was commenced by starting the flow of ultrahigh purity $\mathrm{O}_{2} \cdot 0.1-0.2 \mathrm{ml}$ aliquots of the reaction mixture were removed with a syringe at different time intervals, diluted with anhydrous THF and frozen until analysis. At completion of the run, the mixture was filtered immediately using a $0.2 \mu \mathrm{m}$ syringe filter. GC analysis was with a Agilent 6890 GC equipped with a FID and a Agilent J and W DB-624 capillary column $(30 \mathrm{~m} \times 0.25 \mathrm{~mm} \times 0.25 \mu \mathrm{m})$. GC-MS spectrometry (Agilent GC-7890 A, MS-5975). and ${ }^{1} \mathrm{H}$ and ${ }^{13} \mathrm{C}$ NMR were used to assist product identification. In particular, cyclooctene hydroperoxide was detected using ${ }^{1} \mathrm{H}$ NMR (Supplementary Fig. 17). GC sensitivity factors of the different products were calibrated with commercially available standards when possible. Cyclooctanol and cyclooctanone were used as substitutes for cyclooctenol and cyclooctenone.

Conversion and selectivity data were calculated using GC area ratios of molecules of interest to the internal standard decane, after correction for evaporative loss of COE. The latter was estimated from measurements made with cyclooctane (Supplementary Fig. 2), which has a similar boiling point as COE (Supplementary Table 8) and is non-reactive in air, and taking into account decreasing mole fraction with conversion. Procedural details and equations used for correction for evaporation, conversion and selectivity are in Supplementary Methods Section 1.4.3.

Titration for peroxides. Peroxide test paper (0.5-25 p.p.m. range) was used to test for total destruction of peroxide during titration with triphenylphosphine ( $5 \mathrm{uM}$ in dichloromethane or ethanol). Since peroxide test paper may not be sensitive to polymeric hydroperoxide, KI and starch test was also used to verify the absence of polymeric hydroperoxide.

Data availability. All data are available from the authors upon reasonable request.

\section{References}

1. Kumar, S. \& Jin, R. On the optical absorption properties of quantum-sized gold nanoclusters. J. Nanosci. Lett. 3, 22/21-22/25 (2013).

2. Vargas, A., Santarossa, G., Iannuzzi, M. \& Baiker, A. Fluxionality of gold nanoparticles investigated by Born-Oppenheimer molecular dynamics. Phys. Rev. B Cond. Matter Mater. Phys. 80, 195421 (2009).

3. Cuong, N. T., Sugiyama, A., Fujiwara, A., Mitani, T. \& Chi, D. H. Density functional study of Pt4 clusters adsorbed on a carbon nanotube support. Phys. Rev. B Cond. Matter Mater. Phys. 79, 235417 (2009).

4. Garzon, I. L. et al. Chirality, defects, and disorder in gold clusters. Eur. Phys. J. D Atom. Mol. Opt. Phys. 24, 105-109 (2003).

5. Hayashi, T., Tanaka, K. \& Haruta, M. Selective vapor-phase epoxidation of propylene over $\mathrm{Au} / \mathrm{TiO} 2$ catalysts in the presence of oxygen and hydrogen. J. Catal. 178, 566-575 (1998).

6. Chen, L.-Y., Wang, C.-W., Yuan, Z. \& Chang, H.-T. Fluorescent gold nanoclusters: recent advances in sensing and imaging. Anal. Chem. 87, 216-229 (2015)
7. Ellis, P. R. et al. The cluster beam route to model catalysts and beyond. Farad. Discuss. 188, 39-56 (2016).

8. Zhu, Y., Qian, H., Zhu, M. \& Jin, R. Thiolate-protected Au nanoclusters as catalysts for selective oxidation and hydrogenation processes. Adv. Mater. (Weinheim, Germany) 22, 1915-1920 (2010).

9. $\mathrm{Xu}$, S. et al. Generation of monodisperse particles by using microfluidics: control over size, shape, and composition. Angew. Chem. Int. Ed. Engl. 44, 724-728 (2005).

10. Monnier, J. R. The direct epoxidation of higher olefins using molecular oxygen. Appl. Catal. A Gen. 221, 73-91 (2001).

11. Bawaked, S., Dummer, N. F., Bethell, D., Knight, D. W. \& Hutchings, G. J. Solvent-free selective epoxidation of cyclooctene using supported gold catalysts: an investigation of catalyst re-use. Green Chem. 13, 127-134 (2011).

12. Mishra, S., Mendez, V., Jeanneau, E., Caps, V. \& Daniele, S. A single source precursor route to group 13 homo- and heterometallic oxides as highly active supports for gold-catalyzed aerobic epoxidation of trans-stilbene. Eur. J. Inorg. Chem. 2013, 500-510 (2013)

13. Turner, M. et al. Selective oxidation with dioxygen by gold nanoparticle catalysts derived from 55-atom clusters. Nature 454, 981-983 (2008).

14. Wang, L. et al. Positively charged bulk Au particles as an efficient catalyst for oxidation of styrene with molecular oxygen. Chem. Commun. (Camb.) 49, 3449-3451 (2013).

15. Donoeva, B. G., Ovoshchnikov, D. S. \& Golovko, V. B. Establishing a Au nanoparticle size effect in the oxidation of cyclohexene using gradually changing Au catalysts. ACS Catal. 3, 2986-2991 (2013).

16. Hughes, M. D. et al. Tunable gold catalysts for selective hydrocarbon oxidation under mild conditions. Nature 437, 1132-1135 (2005).

17. Alshammari, H. et al. Initiator-free hydrocarbon oxidation using supported gold nanoparticles. Catal. Sci. Technol. 4, 908-911 (2014).

18. Komiya, S. \& Kochi, J. K. Studies of cationic gold(I) complexes: preparation of tris(trans-cyclooctene)gold trifluoromethanesulfonate. J. Organomet. Chem. 135, 65-72 (1977)

19. Shul'pin, G. B. \& Kozlov, Y. N. Kinetics and mechanism of alkanehydroperoxidation with tert-butyl hydroperoxide catalysed by a vanadate anion. Org. Biomol. Chem. 1, 2303-2306 (2003).

20. Sneeringer, P. V. \& Stenberg, V. I. Quantitative determination of hydroperoxides, alcohols, and ketones in hydrocarbon solvents. Anal. Lett. 4, 485-490 (1971).

21. Zheng, J., Zhang, C. \& Dickson, R. M. Highly fluorescent, water-soluble, sizetunable gold quantum dots. Phys. Rev. Lett. 93, 077402 (2004).

22. Mahajani, S. M., Sharma, M. M. \& Sridhar, T. Uncatalyzed oxidation of cyclohexene. Chem. Eng. Sci. 54, 3967-3976 (1999).

23. Van Sickle, D. E., Mayo, F. R. \& Arluck, R. M. Liquid-phase oxidations of cyclic alkenes. J. Am. Chem. Soc. 87, 4824-4832 (1965).

24. Wu, P., Bai, P., Yan, Z. \& Zhao, G. X. S. Gold nanoparticles supported on mesoporous silica: origin of high activity and role of Au NPs in selective oxidation of cyclohexane. Sci. Rep. 6, 18817 (2016).

25. Liu, Y., Tsunoyama, H., Akita, T., Xie, S. \& Tsukuda, T. Aerobic oxidation of cyclohexane catalyzed by size-controlled Au clusters on hydroxyapatite: size effect in the sub-2 nm regime. ACS Catal. 1, 2-6 (2011).

26. Corma, A. et al. Exceptional oxidation activity with size-controlled supported gold clusters of low atomicity. Nat. Chem. 5, 775-781 (2013).

27. Gajan, D. et al. Gold nanoparticles supported on passivated silica: access to an efficient aerobic epoxidation catalyst and the intrinsic oxidation activity of gold. J. Am. Chem. Soc. 131, 14667-14669 (2009).

28. $\mathrm{Zhu}, \mathrm{H}$. et al. Low-temperature $\mathrm{CO}$ oxidation on $\mathrm{Au} /$ fumed $\mathrm{SiO}_{2}$-based catalysts prepared from $\mathrm{Au}(\mathrm{en})_{2} \mathrm{Cl}_{3}$ precursor. Appl. Catal. A General. 326, 89-99 (2007).

29. Wang, Z. et al. Amine-functionalized siloxane oligomer facilitated synthesis of subnanometer colloidal Au particles. J. Mater. Chem. A Mater. Energy Sustain. 3, 1743-1751 (2015).

\section{Acknowledgements}

The authors acknowledge support of this work by the US Department of Energy, Office of Science, Office of Basic Energy Sciences under Award Number DOE DE-FG02-03ER15457, Y.Y. Wu for a sample of $\mathrm{Au} / \mathrm{SiO}_{2}$, and Anyang Peng for experimental assistance, China Scholarship Council for support of L.Q. and Z.W., CAPES Foundation (Brazil) for support of H.J.d.S., the National Science Foundation (CHE-1465057) for support of STEM work (J.L.), and use of facilities in the John M. Cowley Center for High Resolution Electron Microscopy at Arizona State University.

\section{Author contributions}

Z.W. and L.Q. collected most of the data and participated in interpretation of data and planning of experiments. E.V.B. performed NMR measurements and helped interpretation of all data, J.L. collected all aberration correction TEM images, H.J.d.S prepared some of the catalysts and collected data with them, T.L. helped supervise Z.W. and M.d.C.R. helped supervised H.J.d.S., M.C.K. and H.H.K. provided overall supervision and set direction of the project. 


\section{Additional information}

Supplementary Information accompanies this paper at http://www.nature.com/ naturecommunications

Competing interests: The authors declare no competing financial interests.

Reprints and permission information is available online at http://npg.nature.com/ reprintsandpermissions/

How to cite this article: Qian, L. et al. Stable and solubilized active Au atom clusters for selective epoxidation of cis-cyclooctene with molecular oxygen. Nat. Commun. 8, 14881 doi: 10.1038/ncomms14881 (2017).
Publisher's note: Springer Nature remains neutral with regard to jurisdictional claims in published maps and institutional affiliations.

(c) (i) This work is licensed under a Creative Commons Attribution 4.0 International License. The images or other third party material in this article are included in the article's Creative Commons license, unless indicated otherwise in the credit line; if the material is not included under the Creative Commons license, users will need to obtain permission from the license holder to reproduce the material. To view a copy of this license, visit http://creativecommons.org/licenses/by/4.0/

(C) The Author(s) 2017 


\section{Erratum: Stable and solubilized active Au atom clusters for selective epoxidation of cis-cyclooctene with molecular oxygen}

Linping Qian, Zhen Wang, Evgeny V. Beletskiy, Jingyue Liu, Haroldo J. dos Santos, Tiehu Li, Maria do C. Rangel, Mayfair C. Kung \& Harold H. Kung

Nature Communications 8:14881 doi: 10.1038/ncomms14881 (2017); Published 28 Mar 2017; Updated 11 Aug 2017

The original version of this Article contained an error in which the second affiliation 'Chemical and Biological Engineering Department, Northwestern University, Evanston, Illinois 60208, USA'; was inadvertently switched with the third affiliation 'School of Materials Science and Engineering, Northwestern Polytechnical University, Xi'an, Shaanxi 710072, China'.

Additionally, 'Shaanxi' was misspelt as 'Shaansi'. This has now been corrected in both the PDF and HTML versions of the Article.

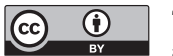

This work is licensed under a Creative Commons Attribution 4.0 International License. The images or other third party material in this article are included in the article's Creative Commons license, unless indicated otherwise in the credit line; if the material is not included under the Creative Commons license, users will need to obtain permission from the license holder to reproduce the material. To view a copy of this license, visit http://creativecommons.org/licenses/by/4.0/

(C) The Author(s) 2017 